\title{
The Value of Information in Energy Harvesting Sensor Networks
}

\author{
Kishor Patil and Dieter Fiems* \\ Ghent University, Dep. of Telecommunications and Information Processing \\ St-Pietersnieuwstraat 41, 9000 Gent, Belgium
}

\begin{abstract}
We study optimal data collection in energy harvesting sensor networks. The sensed data-stream constitutes a sequence of stationary ergodic random variables and, assuming that timely data is valued more, the value of the data is discounted over time such that frequent collection is required to avoid a loss in value. However, unlimited data collection is constrained by both the cost of data collection and the availability of energy.
\end{abstract}

Keywords: energy harvesting, sensor network, performance evaluation

\section{Introduction}

As the key constituent of the Internet of Things (IoT), wireless sensor networks (WSNs) have attracted considerable research interest. Sensor networks collect and monitor spatially distributed data like temperature, humidity, movement and noise [1] and have a variety of applications including military, environmental, home and health applications [2,3]. Sensor networks enable fast data-to-decision applications that act in real time on the collected data, the value the information brings to the decision not only depending on the quality but also on the timeliness of the information. Therefore, analogous to Quality of Service which measures the performance of a data communications network, the term "Quality of Information" (QoI) has been introduced to evaluate performance of sensor networks [4, 5].

In this letter, we study the optimal data collection rate for the hybrid WSN of Zhou et al. [6] which consists of static sensors responsible for sensing environmental variables, and mobile sensors called IoT mobile sinks that move to designated sink locations where they gather the data that is sensed by the static sensors. In particular, we investigate the QoI collected by the IoT mobile sink from a single static energy-harvesting sensor. Energy harvesting sensor nodes mitigate their dependence on batteries by harvesting energy (solar, wind, heat, etc) from their environment [7]. The sensor node under consideration operates energy neutral which means that all energy for sensing and transmissions is harvested, a small on-board battery providing for temporary energy storage. As the node solely depends on harvested energy, one needs to account for the possibility of temporarily running out of energy. Therefore, the sensor node at hand can only transmit when the IoT mobile sink is in range and the sensor has sufficient energy for transmitting.

\section{Analytic model}

We consider an energy harvesting sensor node operating in discrete time. That is, time is divided into fixed length intervals or slots and all transmissions are synchronised with respect to slot boundaries. The sensor node is equipped with on-board memory to store sensed information and a battery for storing harvested energy. For ease of analysis, we discretise the battery levels: the battery can store up to $C$ discrete units (or chunks) of energy.

Let $H_{n}$ denote the number of energy chunks that are harvested during slot $n . H_{n}$ only includes the energy that is available for transmissions. That is, accounting for any conversion loss, and assuming that the node can constantly harvest sufficient energy for sensing, $H_{n}$ is the excess energy that can be used for transmissions. The amount of energy provided by a single chunk corresponds to $1 / N$ th of the energy required to make a single transmission which is assumed to be constant and independent of the value of the information. The choice of a large $N$ corresponds to a fine-grained battery model. However, the same battery capacity then corresponds to more energy chunks $(C$ is larger) which implies that the performance analysis is more computationally demanding (cfr. infra). The sequence $H_{n}$

\footnotetext{
*corresponding author (Email: Dieter.Fiems@UGent.be)
} 
constitutes a sequence of independent and identically distributed random variables, taking values in $\mathbb{N}$. Let $h_{k}$ denote the probability that $k$ energy chunks arrive in a slot, and let $\bar{H}_{k}=\sum_{\ell \geq k} h_{\ell}$ be the corresponding tail distribution.

The value of the sensed data during slot $n$ is denoted by $S_{n}$. We assume that the sequence $S_{n}$ is stationary ergodic; let $\bar{S}=\mathrm{E}\left[S_{0}\right]<\infty$ and let $\sigma_{m}=\mathrm{E}\left[S_{0} S_{m}\right]<\infty$ for $m \in \mathbb{N}$.

We now study the value of information of the sensed data carried by the sensor node at the start of the $n$th slot and denote this value by $V_{n}$. This value is additive and discounted over time with discount factor $\alpha<1$. Discounting is proposed to account for the timeliness of data, older data being less valued. Introducing the variable $T_{n}$ that equals 1 if there is a transmission during slot $n$ and 0 otherwise, the values $V_{n}$ and $V_{n+1}$ relate as,

$$
V_{n+1}=\alpha V_{n} \bar{T}_{n}+S_{n}
$$

with $\bar{T}_{n}=1-T_{n}$. The recursion above implies that data sensed during slot $n$ cannot be transmitted during slot $n$.

The battery level at the start of slot $n$ is denoted by $B_{n}$. As a transmission requires $N$ energy chunks, $H_{n}$ chunks are harvested during slot $n$ and at most $C$ energy chunks can be stored in the battery, we have,

$$
B_{n+1}=\min \left(B_{n}-N T_{n}+H_{n}, C\right) .
$$

It now only remains to express $T_{n}$ in terms of the sequence of transmission opportunities. Let $P_{n}$ be the indicator that there is such an opportunity during slot $n$. The consecutive $P_{n}$ constitute a sequence of independent Bernoulli random variables with mean $\mathrm{E}\left[P_{n}\right]=\mathrm{P}\left[P_{n}=1\right]=p ; p$ is referred to as the collection probability. Assuming the sensor node cannot evaluate the value of information of its data, it always transmits if sufficient energy is available and there is a transmission opportunity. We therefore have,

$$
T_{n}=P_{n} 1_{\left\{B_{n} \geq N\right\}}
$$

where $1_{\{\cdot\}}$ denotes the indicator function which equals 1 if its argument is true and zero if this is not the case.

\section{Analysis}

The set of recursions (1)-(3) now allows for determining the first two moments of the value of the information collected by the IoT mobile sink. We first investigate the existence of a stationary solution $\left(V_{n}^{*}, B_{n}^{*}\right)$ of these recursions.

\subsection{Stability}

The evolution of the battery level does not depend on the value of information. Substituting (3) into (2) shows that for $h_{0}<1, h_{1}>0$ and $0<p<1$, the sequence $B_{n}$ constitutes an ergodic unichain with finite state space $\{0, \ldots, C\}$. Hence, there exist a stationary process $\left\{B_{n}^{\star}, n \in \mathbb{Z}\right\}$ that adheres the recursion (2). Let $\bar{T}_{n}^{\star}=1-P_{n} 1_{\left\{B_{n}^{\star} \geq N\right\}}$ be the corresponding stationary indicator of having no transmission. We now define the stationary value of information process $V_{n}^{*}$ as follows,

$$
V_{n}^{*}=\sum_{k=1}^{\infty} S_{n-k} \alpha^{k-1} \prod_{\ell=1}^{k-1} \bar{T}_{n-\ell}^{*}
$$

The stationarity of $\left\{V_{n}^{*}, n \in \mathbb{Z}\right\}$ follows from the stationarity of $\left\{\left(S_{n}, B_{n}^{*}\right), n \in \mathbb{Z}\right\}$. We now show that (i) the sum on the right-hand side of (4) converges to a random variable with finite mean and (ii) that $\left|V_{n}-V_{n}^{*}\right| \rightarrow 0$ for $n \rightarrow \infty$ for any initial value $V_{0}$. To this end, consider the partial sums $\phi_{m}=\sum_{k=1}^{m} S_{n-k} \alpha^{k-1}$, such that the sequence $\left\{\phi_{m}-\sum_{k=1}^{m} \bar{S} \alpha^{k-1}, m \in \mathbb{N}\right\}$ constitutes an $\mathcal{L}_{1}$-martingale with respect to the filtration $\mathcal{F}_{m}=\sigma\left(S_{n-1}, \ldots, S_{n-m}\right)$. The limit $\phi_{\infty}-\bar{S} /(1-\alpha)$ is then finite with probability 1 (w.p.1) by the martingale convergence theorem, while the finiteness of $\mathrm{E}\left[\phi_{\infty}\right]$ follows from the monotone convergence theorem. As $\phi_{\infty}$ is an upper bound for the sum on the right-hand side of (4), we find that this sum converges to a random variable with finite mean.

Now consider again the process $\left(B_{n}, V_{n}\right)$, starting at $n=0$ with given initial values $\left(B_{0}, V_{0}\right)$. By repeated application of (1), we can write $V_{n}$ in terms of $S_{n}$ and $T_{n}$ as follows,

$$
V_{n}=\alpha^{n} V_{0} \prod_{\ell=1}^{n} \bar{T}_{n-\ell}+\sum_{k=1}^{n} S_{n-k} \prod_{\ell=1}^{k-1} \bar{T}_{n-\ell} .
$$


As $B_{n}$ is an ergodic unichain, we have $\left|B_{n}^{*}-B_{n}\right| \rightarrow 0$ for $n \rightarrow \infty$. Moreover, we have $\left|V_{n}-V_{n}^{*}\right| \rightarrow 0$ for $n \rightarrow \infty$. Indeed, by plugging in (4) and (5) into $\left|V_{n}^{*}-V_{n}\right|$, we have,

$$
\begin{aligned}
\left|V_{n}^{*}-V_{n}\right| & =\left|\sum_{k=1}^{\infty} S_{n-k} \alpha^{k-1} \prod_{\ell=1}^{k-1} \bar{T}_{n-\ell}^{*}-\alpha^{n} V_{0} \prod_{\ell=1}^{n} \bar{T}_{n-\ell}-\sum_{k=1}^{n} S_{n-k} \prod_{\ell=1}^{k-1} \bar{T}_{n-\ell}\right| \\
& \leq \alpha^{n} \sum_{k=1}^{\infty} S_{-k} \alpha^{k-1}+\alpha^{n} V_{0}+\sum_{k=1}^{n} S_{n-k} \alpha^{k-1}\left|\prod_{\ell=1}^{k-1} \bar{T}_{n-\ell}^{*}-\prod_{\ell=1}^{k-1} \bar{T}_{n-\ell}\right| .
\end{aligned}
$$

Consider the last bound. The sum in the first term is finite w.p.1, hence as $\alpha<1$, the first two terms converge to 0 w.p. 1 for $n \rightarrow \infty$. For the third term, consider the random variable $\tau=\inf \left\{n ; T_{n}=1\right.$ and $B_{m}=B_{m}^{*}$ for $\left.m \geq n\right\}$ which is finite w.p.1. Then for $n>\tau$, one easily verifies that the third term is 0 as well. As $\lim _{n \rightarrow \infty} \mathrm{P}[\tau \geq n]=0$, we conclude that the third term converges to 0 as well.

Summarising, we have shown that there exists a stationary ergodic process $\left(B_{n}^{*}, V_{n}^{*}\right)$, with $V_{n}^{*}$ given by (4), adhering the recursions (1)-(3). Moreover, whatever the initial condition $\left(B_{0}, V_{0}\right),\left|B_{n}^{*}-B_{n}\right| \rightarrow 0$ and $\left|V_{n}^{*}-V_{n}\right| \rightarrow 0$, for $n \rightarrow \infty$.

\subsection{Moments}

We focus on the first two moments of the stationary process $V_{n}^{*}$. Let $b_{k}=\mathrm{P}\left[B_{n}^{*}=k\right]$ be the stationary probability of having battery level $k$ and let $v_{k}=\mathrm{E}\left[V_{n}^{*} 1_{\left\{B_{n}^{*}=k\right\}}\right]$ be the mean value of information at the sensor node for battery level $k$. By the equations for $V_{n}, B_{n}$ and $T_{n}$ and by conditioning on the battery level and the availability of a transmission opportunity in the preceding slot, we find that the mean value of the information at battery level $k$ adheres,

$$
v_{k}=\alpha \sum_{\ell=0}^{C} v_{\ell} h_{k, \ell}\left(1-p 1_{\{\ell \geq N\}}\right)+\bar{S} b_{k}
$$

for $k=0,1, \ldots, C$, where we introduced $h_{k, \ell}=1_{\{k<C\}} h_{k-\ell}+1_{\{k=C\}} \bar{H}_{C-\ell}$ to simplify notation.

Again by conditioning on the battery level and the availability of a transmission opportunity in the preceding slot, the battery level probabilities adhere,

$$
b_{k}=\sum_{\ell=0}^{C} b_{\ell}\left(h_{k, \ell}+p\left(h_{k, \ell-N}-h_{k, \ell}\right) 1_{\{\ell \geq N\}}\right)
$$

for $k=0,1, \ldots, C$. We now introduce the column vectors $\mathbf{v}=\left[v_{k}\right]_{k=0}^{C}$ and $\mathbf{b}=\left[b_{k}\right]_{k=0}^{C}$, as well as the following matrices,

$$
\mathcal{A}=\left[h_{k, \ell}\left(1-p 1_{\{\ell \geq N\}}\right)\right]_{k, \ell=0}^{C}, \quad \mathcal{B}=\left[h_{k, \ell}+p\left(h_{k, \ell-N}-h_{k, \ell}\right) 1_{\{\ell \geq N\}}\right]_{k, \ell=0}^{C} .
$$

The set of equations (6)-(7) is then equivalent to $\mathbf{v}=\alpha \mathcal{A} \mathbf{v}+\bar{S} \mathbf{b}$ and $\mathbf{b}=\mathcal{B} \mathbf{b}$, such that, accounting for the normalisation condition $\mathbf{e}^{T} \mathbf{b}=1$, we find,

$$
\mathbf{b}=\left(\mathcal{B}-\mathcal{I}+\mathbf{e e}^{T}\right)^{-1} \mathbf{e}, \quad \mathbf{v}=\bar{S}(\mathcal{I}-\alpha \mathcal{A})^{-1} \mathbf{b} .
$$

Here $\mathbf{e}$ is a column vector of ones, the superscript $T$ indicates the matrix transpose, and $\mathcal{I}$ denotes the identity matrix.

We can then express the mean value of the sensed data per time slot that is actually collected in terms of the $v_{k}$ 's as follows,

$$
\bar{V}=\mathrm{E}\left[V_{n} 1\left\{P_{n}=1, B_{n} \geq N\right\}\right]=p \sum_{k=N}^{C} v_{k} .
$$

Indeed, there are only transmissions if the battery level exceeds the threshold and there is a transmission opportunity.

The calculation of the second moment is considerably more involved. By the system equations (1)-(3), we have,

$$
v_{k}^{(2)}=\mathrm{E}\left[\left(V_{n+1}^{*}\right)^{2} 1_{\left\{B_{n+1}=k\right\}}\right]=\alpha^{2} \sum_{\ell=0}^{C}\left(1-p 1_{\{\ell \geq N\}}\right) h_{k, \ell} v_{\ell}^{(2)}+2 \alpha \sum_{\ell=0}^{C}\left(1-p 1_{\{\ell \geq N\}}\right) h_{k, \ell} \xi_{\ell}^{(0)}+\sigma_{0} b_{k},
$$

for $k=0,1, \ldots, C$, with $\xi_{\ell}^{(0)} \doteq \mathrm{E}\left[V_{n}^{*} S_{n} 1_{\left\{B_{n}^{*}=\ell\right\}}\right]$. This set of equations then corresponds to the following matrix equation,

$$
\mathbf{v}^{(2)}=\alpha^{2} \mathcal{A} \mathbf{v}^{(2)}+2 \alpha \mathcal{A} \xi^{(0)}+\sigma_{0} \mathbf{b}
$$


with $\mathbf{v}^{(2)}=\left[v^{(2)}\right]_{k=0}^{C}$ and $\xi^{(0)}=\left[\xi_{k}^{(0)}\right]_{k=0}^{C}$. To determine the remaining unknown vector $\xi^{(0)}$, let $\xi_{k}^{(m)} \doteq \mathrm{E}\left[V_{n-m}^{*} S_{n} 1_{\left\{B_{n-m}^{*}=k\right\}}\right]$, such that in view of the system equations (1)-(3) we have,

$$
\xi_{k}^{(m)}=\alpha \sum_{\ell=0}^{C}\left(1-p 1_{\{\ell \geq N\}}\right) h_{k-\ell} \xi_{\ell}^{(m+1)}+\sigma_{m+1} b_{k} .
$$

The former equation allows for calculating $\xi_{k}^{(m)}$ recursively. Indeed, defining the vector $\xi^{(m)}=\left[\xi_{k}^{(m)}\right]_{k=0}^{C}$, we have,

$$
\xi^{(m)}=\alpha \mathcal{A} \xi^{(m+1)}+\sigma_{m+1} \mathbf{b} .
$$

Recursively substituting this equation into itself then yields,

$$
\xi^{(0)}=\sum_{\ell=0}^{m-1} \sigma_{\ell+1} \alpha^{\ell} \mathcal{A}^{\ell} \mathbf{b}+\alpha^{m} \mathcal{A}^{m} \xi^{(m)}=\sum_{\ell=0}^{\infty} \sigma_{\ell+1} \alpha^{\ell} \mathcal{A}^{\ell} \mathbf{b} .
$$

where we used the fact that $\alpha^{m} \mathcal{A}^{m} \rightarrow 0$ for $m \rightarrow \infty$ as its largest eigenvalue is less than one.

Summarising, the second order moment vector $\mathbf{v}^{(2)}$ can be written as,

$$
\mathbf{v}^{(2)}=\left(\mathcal{I}-\alpha^{2} \mathcal{A}\right)^{-1}\left(2 \sum_{\ell=0}^{\infty} \sigma_{\ell} \alpha^{\ell} \mathcal{A}^{\ell} \mathbf{b}-\sigma_{0} \mathbf{b}\right) .
$$

The variance of the value of information collected in a slot is then,

$$
\operatorname{var}[V]=\mathrm{E}\left[\left(V_{n}^{*}\right)^{2} 1\left\{P_{n}=1, B_{n}^{*} \geq N\right\}\right]-\bar{V}^{2}=p \sum_{k=N}^{C} v_{k}^{(2)}-\bar{V}^{2} .
$$

\subsection{Extension to Markov modulated energy arrivals}

The assumption that the number of energy chunks that arrive in a slot constitutes a sequence of independent random variables is not essential for the calculations. We here extend the method to allow for correlated arrivals. We limit the discussion to the calculation of the mean value of the sensed data per time slot.

Let $\left\{M_{n}, n \in \mathbb{Z}\right\}$ be an ergodic Markov process with finite state space $\mathcal{M}=\{1, \ldots, M\}$, and let $m_{i j}$ denote the transition probability from state $i$ to state $j$ (for $i, j \in \mathcal{M}$ ). $M_{n}$ is the environment process which modulates the energy arrival process. That is, we assume that the number of energy chunks $H_{n}$ that arrive in slot $n$, depends on $M_{n}$. Such energy arrival processes have been proposed a.o. for energy harvesting body sensor networks in [9] and for solar harvesting in [10].

Let $h_{k}^{(i)}=\mathrm{P}\left[H_{n}=k \mid M_{n}=i\right]$ be the probability that $k$ chunks arrive when the environment is in state $i$. Further let $\bar{H}_{k}^{(i)}=\sum_{\ell \geq k} h_{\ell}^{(i)}$ and let $h_{k, \ell}^{(i)}=1_{\{k<C\}} h_{k-\ell}^{(i)}+1_{\{k=C\}} \bar{H}_{C-\ell}^{(i)}$. We now calculate the joint probability of the environment state and the battery level. Let $b_{k}^{(j)}=\mathrm{P}\left[B_{n}=k, M_{n}=j\right]$, by conditioning on the battery level, the environment state and the availability of a transmission opportunity in the preceding slot, these probabilities adhere,

$$
b_{k}^{(j)}=\sum_{i=1}^{M} m_{i j} \sum_{\ell=0}^{C} b_{\ell}^{(i)}\left(h_{k, \ell}^{(i)}+p\left(h_{k, \ell-N}^{(i)}-h_{k, \ell}^{(i)} 1_{\{\ell \geq N\}}\right),\right.
$$

for $k=0,1, \ldots, C$. The set of equations above, complemented by the normalisation condition $\sum_{k=0}^{C} \sum_{j=1}^{M} b_{k}^{(j)}=1$, allows for determining the probabilities $b_{k}^{(j)}$.

Let $v_{k}^{(j)}=\mathrm{E}\left[V_{n} 1_{\left\{B_{n}=k, M_{n}=j\right\}}\right]$ be the mean value of the information at the sensor node for battery level $k$ and environment state $j$. By conditioning on the battery level, the environment state and the availability of a transmission opportunity in the preceding slot, we find that the mean value of the information at battery level $k(k=0,1, \ldots, C)$ and environment state $j$ adheres,

$$
v_{k}^{(j)}=\alpha \sum_{i=1}^{M} m_{i j} \sum_{\ell=0}^{C} v_{\ell}^{(i)} h_{k, \ell}^{(i)}\left(1-p 1_{\{\ell \geq N\}}\right)+\bar{S} b_{k}^{(j)}
$$

The systems of equations (9)-(10) are again linear, though there are now $M$ equations for every battery state. The mean value of the sensed data per time slot that is actually collected then equals,

$$
\bar{V}=p \sum_{i=1}^{M} \sum_{k=N}^{C} v_{k}^{(i)}
$$




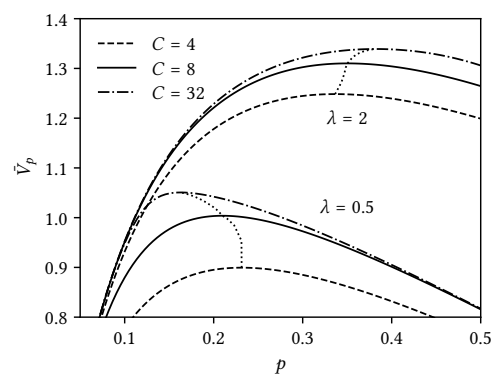

Figure 1: Value of the information $\bar{V}_{p}$ versus $p$ for different $C$ as indicated.

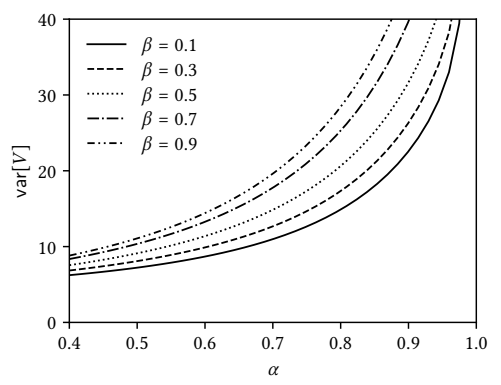

Figure 4: Variance $\operatorname{var}[V]$ versus $\alpha$ for different $\beta$.

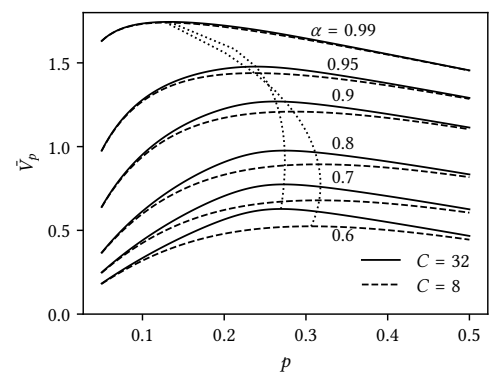

Figure 2: Value of the information $\bar{V}_{p}$ versus $p$ for different $\alpha$ as indicated.

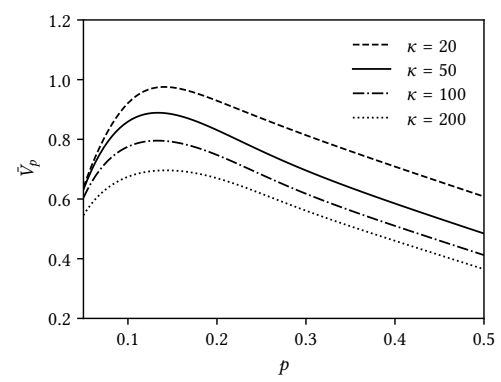

Figure 5: Value of the information $\bar{V}_{p}$ versus $p$ for different $\kappa$.

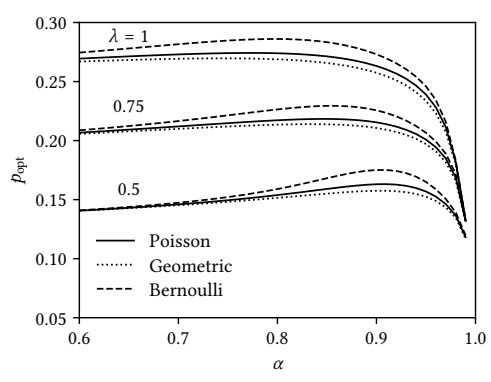

Figure 3: Optimal $p$ versus $\alpha$ for different harvesting distributions.

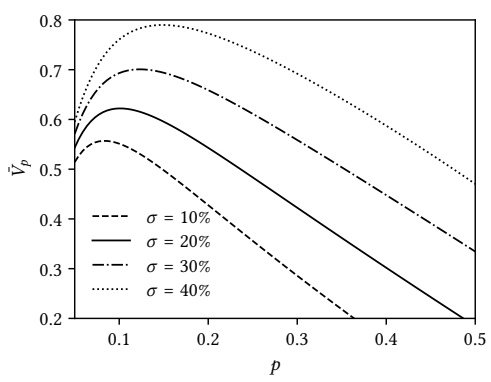

Figure 6: Value of the information $\bar{V}_{p}$ versus $p$ for different $\sigma$.

\section{Discussion and numerical results}

We now investigate the optimal data collection policy for the sensor node at hand. We assume that there is a cost $c$ associated to data collection such that the average value after collection equals $\bar{V}_{p}=-c p+\bar{V}$.

Figures 1 and 2 depict the value of information $\bar{V}_{p}$ in terms of the data collection probability $p$. We assume that the mean value of information sensed in a slot equals twice the cost of collecting it: $c=1$ and $\bar{S}=2$. Moreover, $N=4$ energy chunks are required for transmission and the number of harvested energy chunks is Poisson distributed with mean $\lambda$. Figure 1 fixes the discount factor to $\alpha=0.9$ and shows $\bar{V}_{p}$ for various battery capacities $C \in\{4,8,32\}$ and $\lambda \in\{0.5,2\}$ as indicated. In contrast, Figure 2 fixes the energy arrival rate to $\lambda=1$, and shows the value of information $\bar{V}_{p}$ for various discount factors $\alpha \in\{0.6,0.7,0.8,0.9,0.95,0.99\}$ and battery capacities $C \in\{8,32\}$ as indicated.

If no data is collected, there is neither cost nor data such that the value is zero for $p=0$. As the cost of data collection is limited in the figures, the value of information first increases for increasing $p$ and then decreases again. For high $p$, the chance of insufficient energy increases such that the gain of more frequent data collection cannot compensate the collection cost. Figure 1 and 2 further reveal that increasing the battery size is beneficial, as a larger battery can better compensate periods with little energy harvesting. The marginal gain of increasing the battery capacity however drops quickly. In addition, Figure 1 and 2 show that increasing $\lambda$ and $\alpha$ is beneficial as well. This is also expected as there can be more transmissions if $\lambda$ increases (there is more energy) and there will be more information if $\alpha$ increases.

The dotted lines in Figures 1 and 2 connect the values of the different curves at the optimal collection probability (for fixed $\lambda$ and variable $C$ in Figure 1 and for fixed $C$ and variable $\alpha$ in Figure 2). Figure 1 shows that the optimal collection probability can either increase or decrease for increasing $C$, depending on $\lambda$. For larger $\lambda$, the optimal collection probability increases for increasing $C$. This is what one expects as the chance to have energy for transmission increases when $C$ increases (there is less energy loss). For small $\lambda$, the optimal collection probability decreases for increasing $C$. This can be explained by noting that energy is scarce for small $\lambda$. If $C$ is small as well, even more energy is lost if the information is not frequently collected. When $C$ increases, the energy loss drops and the collection probability can drop as well. For increasing $\alpha$, Figure 2 shows a slight increase followed by a quick decrease of the optimal collection probability. For $\alpha$ large, a drop in $\alpha$ means that the value of information is discounted faster such that we need to collect more. For $\alpha$ small, the cost of collection is high compared to the value at the sensor node. If $\alpha$ increases, this value increases such that we can collect more.

The latter observations are also confirmed by Figure 3 which depicts the optimal collection probability versus $\alpha$ 
for different energy arrival distributions and different values of the mean number of energy chunks $\lambda$ arriving in a slot as indicated. The collection cost is $c=2$, the battery can store $C=32$ energy chunks and the mean value of the sensed data is $\bar{S}=2$. The optimal collection probability is higher for higher $\lambda$ as an increase in $\lambda$ implies that it is less likely that a lack of energy prevents transmissions. Moreover, as an increase in the variance of the energy chunk distribution (the variance of the geometric distribution is higher than that of the Poisson distribution which in turn is higher than that of the Bernoulli distribution) implies that it is more likely to run out of energy, $p_{\text {opt }}$ decreases with the variance. Finally, we note that the optimal collection probability is not sensitive to changes in $\alpha$ for low $\alpha$, while it is very sensitive for high $\alpha$, irrespective of the energy chunk distribution.

Figure 4 illustrates the influence of the correlation in the information process $S_{n}$ on the variance of the value of information. The autocovariance of the information process decays exponentially with rate $\beta: \sigma_{n}=\beta^{n} \operatorname{var}\left[S_{0}\right]+\bar{S}^{2}$. Such a decay can e.g. be found when the information process constitutes an autoregressive process of order 1.

Figure 4 shows the variance of the value of the information at the optimal collection probability versus the discount factor $\alpha$ for $\beta$ as indicated and for $\operatorname{var}\left[S_{0}\right]=16$ and $\bar{S}=2$. The collection cost is $c=2$, the battery can store $C=32$ chunks and the energy arrival distribution is a Poisson distribution with mean $\lambda=1$. The figure shows that for higher $\alpha$ as well as for higher positive correlation in the sensing process, the variance of the value of information increases considerably. While correlation does not affect the mean value of information, it does affect the corresponding variance meaning that the collected value of information is more likely to deviate considerably from its average value.

Finally, we investigate the effect of correlation in the harvesting process. Using the results of section 3.3, Figures 5 and 6 show the value of the information $\bar{V}_{p}$ versus the collection probability $p$ when the harvesting process is an interrupted Poisson process. The harvesting process is either active or inactive, the mean time to remain active and inactive being denoted by $\kappa_{a}$ and $\kappa_{i}$, respectively. Let $\kappa=\kappa_{a}+\kappa_{i}$ denote the mean length of an active-inactive cycle and let $\sigma=\kappa_{a} / \kappa$ denote the fraction of active slots. While active, the number of energy chunks is Poisson distributed with mean $\lambda$, while there is no harvesting during inactive slots. In both Figures, the collection cost is $c=1$, the battery can store $C=32$ energy chunks, the discount factor is $\alpha=0.9$, the mean value of the sensed data is $\bar{S}=2$. and $N=4$ chunks are required per transmission. In Figure 5, we fix $\sigma=50 \%$ and $\lambda=1$ and consider different $\kappa$ as indicated. Large $\kappa$ means that there are long periods with harvesting followed by long periods without harvesting, which clearly affects performance. Moreover, the optimal collection probability decreases for larger $\kappa$ as well, as it is likely that there is no energy during long periods without harvesting. In Figure 6, we fix $\kappa=100$ and choose different $\sigma$ as indicated, while we keep the mean number of energy chunks per slot constant, $\sigma \lambda=1$. Small $\sigma$ means that harvesting is concentrated in a few slots, again followed by many slots without harvesting, and therefore negatively affects performance. For increasing $\sigma$, the optimal collection probability increases as the lengths of the periods without harvesting decrease.

\section{References}

[1] I.F. Akyildiz, W. Su, Y. Sankarasubramaniam, E. Cayirci. Wireless sensor networks: a survey. Computer Networks 38(4):393-422, 2002.

[2] A. Al-Fuqaha, M. Guizani, M. Mohammadi, M. Aledhari, M. Ayyash. Internet of Things: A Survey on Enabling Technologies, Protocols, and Applications. IEEE Communications Surveys and Tutorials 17(4):2347-2376, 2015.

[3] H.M. Ammari, N. Gomes, M. Jacques, B. Maxim, D. Yoon. A Survey of Sensor Network Applications and Architectural Components. Ad Hoc \& Sensor Wireless Networks 25(1-2):1-44, 2015.

[4] V. Sachidananda, A. Khelil, N. Suri. Quality of Information in Wireless Sensor Networks: A Survey. In: Proc. of the 15th International Conference on Information Quality (ICIQ'10). pp. 193-207, 2010.

[5] C. Bisdikian, L.M. Kaplan, M.B. Srivastava. On the quality and value of information in sensor networks. ACM Transactions on Sensor Networks 9(4):48, 2013.

[6] Z. Zhou, C. Du, L. Shu, G. Hancke, J. Niu, H. Ning. An Energy-Balanced Heuristic for Mobile Sink Scheduling in Hybrid WSNs. IEEE Transactions on Industrial Informatics 12(1):28-40, 2016.

[7] I. Ahmed, M.M. Butt, C. Psomas, A. Mohamed, I. Krikidis, M. Guizani. Survey on energy harvesting wireless communications: Challenges and opportunities for radio resource allocation. Computer Networks 88:234-248, 2015.

[8] C. Blondia, O. Casals. Statistical multiplexing of VBR sources: A matrix-analytic approach. Performance Evaluation 16(1):5-20, 1992. 
[9] A. Seyedi, B. Sikdar. Modeling and Analysis of Energy Harvesting Nodes in Body Sensor Networks. In: Proc. of the 5th International Workshop on Wearable and Implantable Body Sensor Networks, 2008.

[10] A.E. Şuşu, A. Acquaviva, D. Atienza, G. De Micheli. Stochastic Modeling and Analysis for Environmentally Powered Wireless Sensor Nodes. In: Proc. of the 6th International Symposium on Modeling and Optimization in Mobile, Ad Hoc, and Wireless Networks and Workshops (WiOPT), 2008. 